\title{
Technical developments for computed tomography on the CENBG nanobeam line
}

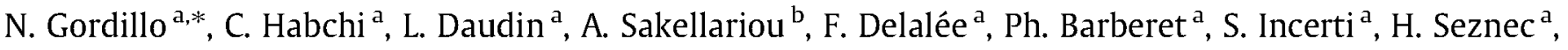 \\ Ph. Moretto ${ }^{a}$ \\ ${ }^{a}$ Université Bordeaux 1, CNRS/IN2P3, Centre d'Etudes Nucléaires de Bordeaux Gradignan, CENBG, Chemin du Solarium, BP120, 33175 Gradignan, France \\ ${ }^{\mathrm{b}}$ Research School of Physical Sciences and Engineering, The Australian National University, Canberra, ACT 0200, Australia
}

\begin{abstract}
A B S T R A C T
The use of ion microbeams as probes for computed tomography has proven to be a powerful tool for the three-dimensional characterization of specimens a few tens of micrometers in size. Compared to other types of probes, the main advantage is that quantitative information about mass density and composition can be obtained directly, using specific reconstruction codes. At the Centre d'Etudes Nucleaires de Bordeaux Gradignan (CENBG), this technique was initially developed for applications in cellular biology. However, the observation of the cell ultrastructure requires a sub-micron resolution. The construction of the nanobeam line at the Applications Interdisciplinaires des Faisceaux d'Ions en Region Aquitaine (AIFIRA) irradiation facility has opened new perspectives for such applications.

The implementation of computed tomography on the nanobeam line of CENBG has required a careful design of the analysis chamber, especially microscopes for precise sample visualization, and detectors for scanning transmission ion microscopy (STIM) and for particle induced X-ray emission (PIXE). The sample can be precisely positioned in the three directions $X, Y, Z$ and a stepper motor coupled to a goniometer ensures the rotational motion. First images of 3D tomography were obtained on a reference sample containing microspheres of certified diameter, showing the good stability of the beam and the sample stage, and the precision of the motion.
\end{abstract}

\section{Introduction}

During the last years the interest for applications in 3D mapping using ion beam as a tomography probe has significantly increased although there are still few laboratories that use these techniques [1-3]. The first experiments using scanning transmission ion microscopy-tomography (STIM-T) and particle induced X-ray emission-tomography (PIXE-T) were done at the end of the eighties and beginning of the nineties, respectively [4,5]. Since then, the development of 3D microscopy techniques providing a spatial resolution less than $1 \mu \mathrm{m}$ has demonstrated to be very suitable in materials research [6] as well as life sciences [1,3,7]. One of the most interesting advantages of this technique is the use of STIM$\mathrm{T}$ in combination with PIXE-T. These two analytical tools in combination with a specific computer algorithm code to reconstruct the data $[8,9]$ allow to analyze the spatial distribution of different elements and also the composition of the sample under study. In addition, the limited angle tomography has been recently implemented by the group of LIPSION [10]. This permits to obtain 3D structural information, from experimental data covering only an angular range of $120^{\circ}$ instead of $180^{\circ}$.
The feasibility of STIM and PIXE tomography at the cell level was previously demonstrated at CENBG on human cancer cells [11] using the microbeam line. The recent installation of the ultra-stable single-ended HVEE 3.5 MV Singletron ${ }^{\mathrm{TM}}$ accelerator at CENBG in the framework of the AIFIRA facility opens the opportunity to focus alpha, proton and deuteron ion beams in the range of $\mathrm{MeV}$ to sub-micrometric scale [12,13] at the nanobeam line (based on a $25 \mathrm{~cm}$ working distance doublet-triplet configuration of $\mathrm{Ox}$ ford Microbeam Ltd. OM-50 ${ }^{\mathrm{TM}}$ quadrupole lenses). The need to go to the nanometric level involves solving some difficulties, like focusing and the stability of the beam, in order to obtain reproducibility in the measurements. With this work, we want to demonstrate that the nanobeam line of CENBG is already capable to perform standard STIM tomography. At the moment, the nanobeam line is currently reaching a resolution of about $300 \mathrm{~nm}$ when working in STIM configuration with a current of a few thousands ions of counts per second and about $1 \mu \mathrm{m}$ when working in PIXE configuration with a current of a few $\mathrm{pA}$ up to hundreds of pA. Such a resolution allows to get promising results at the sub-cellular level.

Technical developments have been carried out on the CENBG nanobeam line to perform a 3D characterization of the specimens with high resolution. In particular, we will emphasize on the 3D tomography setup. Moreover, we will confirm the stability of the goniometer rotation axis during the analysis and the spatial reso- 
lution in these first experiments using the nanobeam line in STIM$\mathrm{T}$ configuration. A whole description of the CENBG nanobeam line can be found in Refs. $[14,15]$.

\section{3D-tomography setup}

The experimental setup has been designed by the CENBG engineers especially for 3D-tomography analysis. The analysis chamber has been equipped with a special goniometer with a four-axis movement designed for this purpose. A picture of the analysis chamber and goniometer is shown in Fig. 1a. A careful adjustment of the sample along the rotation axis is necessary. For this, two micrometric screws permit the adjustment of the tilt angle, and two others are used for displacements in the horizontal plane. Hence, the sample can be aligned with the vertical rotation axis of the goniometer with an accuracy of $1.0^{\circ}$ in tilt and of $1.0 \mu \mathrm{m}$ in translation. The alignment is done manually using the micrometric screws and motor-step in air (Fig. 1b). Next, the goniometer is rotated in order to assure the line-up of the sample with the rotation axis. In the mean time, a movie is recorded using the microscope and the camera placed at the chamber. That movie is used to check for a displacement of the rotation axis during the process of rotating. Finally, if everything is ok, the chamber is closed and evacuated. During the evacuation of the chamber, the sample undergoes a slight misalignment in the range of a few micrometers. Smaller corrections in the horizontal plane positioning can be done, directly in vacuum. The goniometer can rotate with an angular step size of $0.01^{\circ}$. For a precise tracking of the sample, the chamber is equipped with three microscope objectives with different magnifications: one $10 \times$ on the front side, i.e. in the direction of the incoming beam, one $4 \times$ and one $20 \times$ viewing the back side of the sample. The STIM detector, Faraday cup (FC) and microscope objectives ( $4 \times$ and $20 \times$ ) are placed at $0^{\circ}$ in the forward direction on a motorized stage, which can be remote-controlled (Newport ${ }^{\mathrm{TM}}$ ) from the experimental room, to position them conveniently in the beam path. An X-ray detector can be placed in the backward direction at $45^{\circ}$ on the incoming beam axis.

\subsection{Reference sample preparation}

A reference sample composed of borosilicate glass microspheres was prepared for the first experiment. These microspheres were chosen as a model for STIM-T study due to their regular spherical shape, certified diameter of $5.6 \pm 0.5 \mu \mathrm{m}$ and relatively low mass

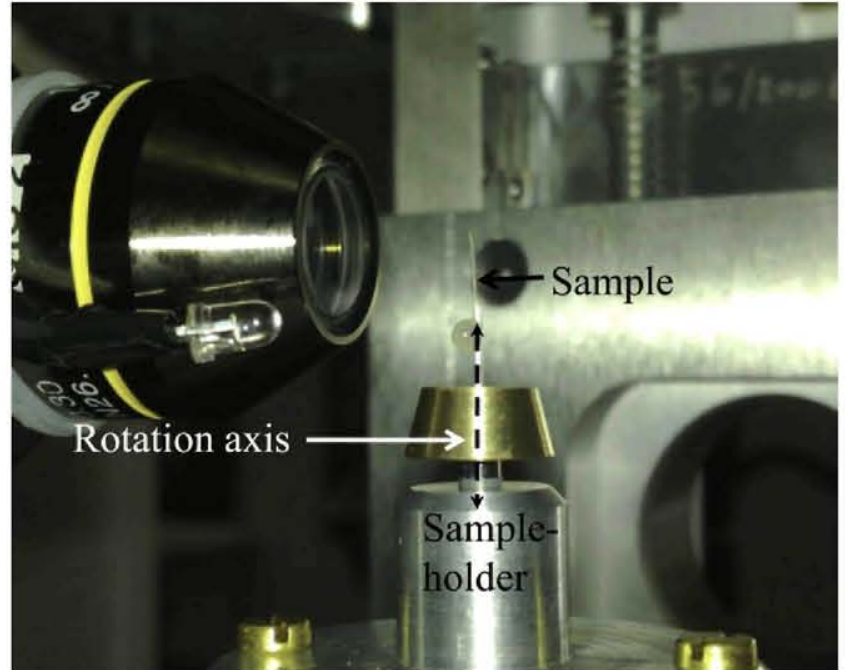

Fig. 2. Reference sample mounted on the goniometer sample holder. The dashed line indicates the vertical rotation axis. The $10 \times$ microscope objective is also shown.

density of $2.5 \pm 0.5 \mathrm{~g} / \mathrm{cm}^{3}$ [16]. Although the size of these microspheres does not reach sub-micron level, they are suitable for a first test of the spatial resolution in STIM-T. A suitable concentration of microspheres can be achieved by mixing $90.0 \pm 0.2 \mathrm{mg}$ of them in $1.0 \pm 0.1 \mathrm{ml}$ of a liquid polymer (formvar). Then, the fibers were stretched out and dried in air for 1 day and glued on top of a metallic needle $500 \mu \mathrm{m}$ in diameter. The length of the polymer fiber was $5 \mathrm{~mm}$. The sample mounted on the goniometer sample holder is shown in Fig. 2. The sample was aligned along the vertical axis in air and then in vacuum, according to the procedure described previously.

\subsection{Experimental conditions}

For this experiment, a $3 \mathrm{MeV} \mathrm{H} \mathrm{H}^{+}$beam was used as a probe. STIM-T requires a good definition of the beam spot on the sample. For that reason, a circular collimator of $5 \mu \mathrm{m}$ in diameter is used as an object for the quintuplet configuration. It is followed by a circular aperture of $20 \mu \mathrm{m}$ in diameter placed before the triplet, $8.25 \mathrm{~m}$ away from the first collimator, in order to keep aberrations low. With these settings, the beam size in the focal plane is around

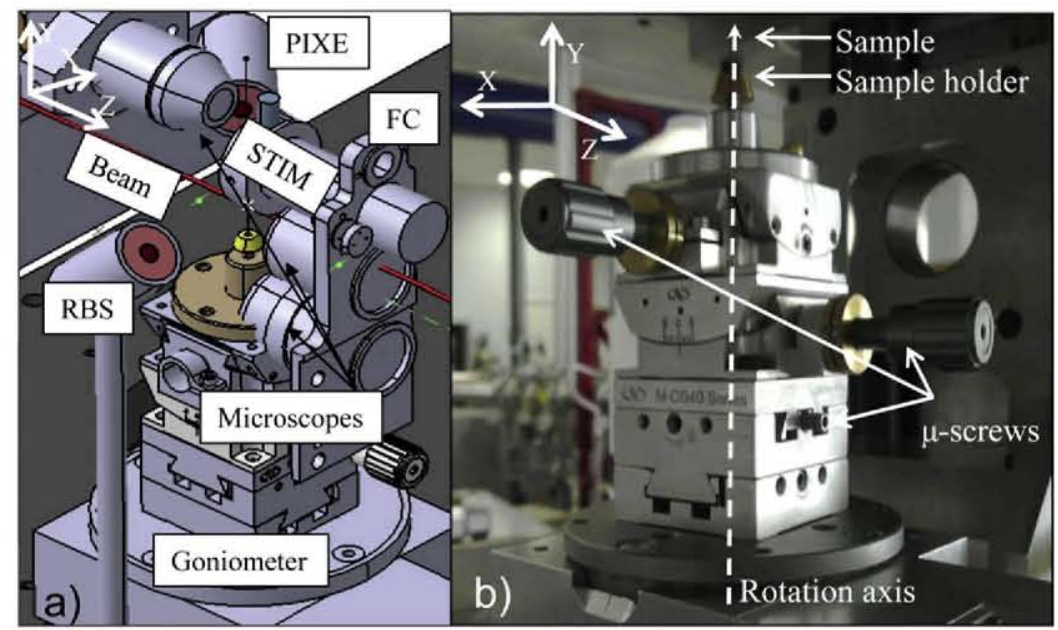

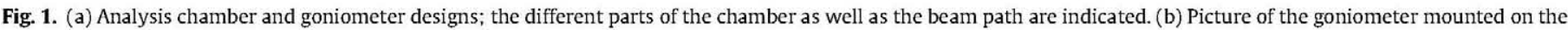
XYZ motorized stage. The dashed line indicates the vertical rotation axis. The sample, sample holder and micrometric screws are also shown. 

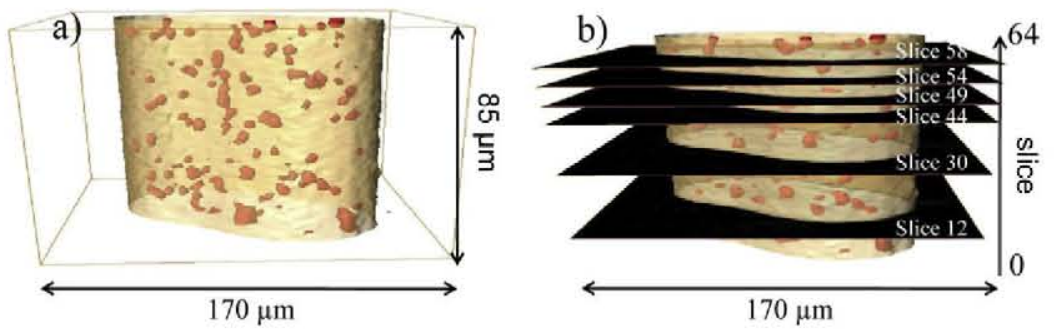

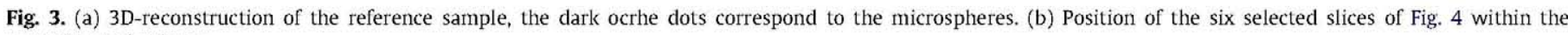
reconstructed volume.
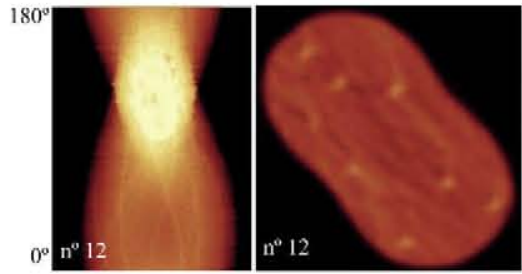

$180^{\circ}$
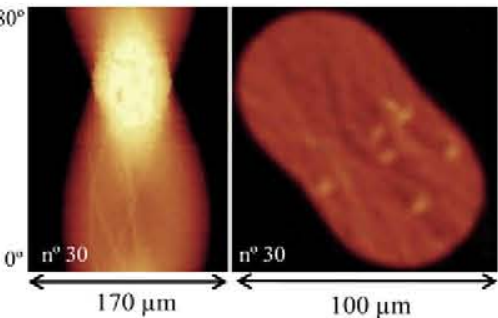

$100 \mu \mathrm{m}$
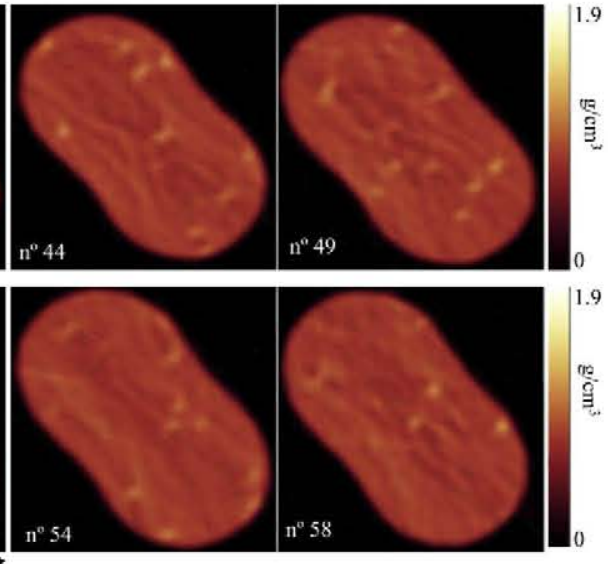

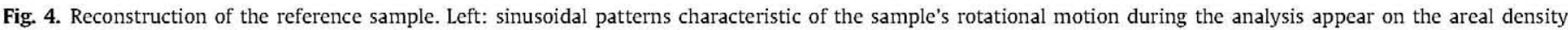

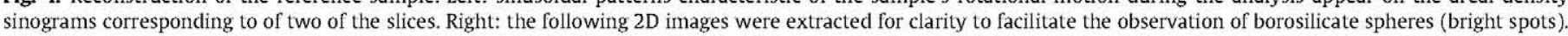

$250 \mathrm{~nm}$ in diameter. The current was kept low enough (less than 2000 ions/s) to avoid any damage of the sample during the analysis. In these conditions, STIM-T is considered as a non-destructive technique, even avoiding any shrinkage of the sample. The transmitted ions are collected in a passivated implanted planar silicon detector (Canberra PIPS detector, $25 \mathrm{~mm}^{2}, 12 \mathrm{keV}$ energy resolution), placed at $0^{\circ}$ on the incoming beam axis. The detector was about $6 \mathrm{~mm}$ away from the sample and, was moved mechanically during the acquisition. Thus we avoid any overexposure in the area of detection. The data acquisition and the 3D-scan analysis are fully automated. The beam scan over the region under study is programmed at the beginning of the experiment. In this case, a $170 \times 85 \mu \mathrm{m}^{2}$ area was selected for 3D STIM-T with a total of 64 horizontal slices, obtained from a $128 \times 64$ pixel scan. So, the resolution of the scan was $1.33 \mu \mathrm{m} /$ pixel. With the purpose of avoiding sample damage, several rapid scans were performed for every projection. The scan speed was here selected to be $100 \mu \mathrm{s} /$ pixel. A total of 100 projections were recorded over $180^{\circ}$ angular range with steps of $1.8^{\circ}$, with using 50 scans/projection. The total duration of the analysis was typically around $2 \mathrm{~h}$.

\section{Data analysis: 3D-reconstruction}

For data treatment the TomoRebuild data reduction software was used. This program, developed at CENBG, was designed to be interactive, simple to use and easily modifiable according to experimental conditions. The program interface consists of a single window, allowing reconstruction of $2 \mathrm{D}$ or $3 \mathrm{D}$ tomography images. Intermediate results can be easily accessed to be checked and/or modified at different steps of the reconstruction. Automatic correction procedures can be implemented to improve noisy data. The reconstruction is based on the filtered backprojection algorithm. A detailed description of how the TomoRebuild software operates can be found in Ref. [17].

\section{Results and discussion}

The 3D-reconstruction of the sample is shown in Fig. 3a. Two isosurfaces are displayed. The density closed to zero is characteristic of the limit between the sample and surrounding vacuum (light ochre). The highest densities show the glass microspheres (dark ochre). Their spherical shape is visible in the slices (bright spots in Fig. 4), selected along the fiber as indicated in Fig. 3b. The areal density calculated for two of those slices ( $n^{\circ} 12$ and $n^{\circ} 30$ ) are displayed in the sinograms in Fig. 4 (left). A linear scale ranging from zero (black) to the highest areal density $1.9 \mathrm{~g} / \mathrm{cm}^{3}$ (white) was used to represent the data. The reconstruction was performed assuming a uniform theoretical composition of formvar $\mathrm{C}_{3} \mathrm{H}_{8} \mathrm{O}$ for all the volume. Because of the approximation made on sample composition, the reconstructed values of the mass density given hereafter are only indicative, and should not be considered as a precise determination. The reconstructed density of formvar was $1.09 \pm 0.01 \mathrm{~g} / \mathrm{cm}^{3}$ in good agreement with the expected density $1.2 \mathrm{~g} / \mathrm{cm}^{3}$. The mass density obtained for the glass microspheres $1.51 \pm 0.07 \mathrm{~g} / \mathrm{cm}^{3}$ was lower than the certified $2.5 \pm 0.5 \mathrm{~g} / \mathrm{cm}^{3}$. The measured diameter of the spheres was around $5 \mu \mathrm{m}$ which is in a good agreement with the certified one.

In the present study, the sinogram is composed of 100 horizontal lines (one per projection). The characteristic shape of the sinusoidal paths, showing a common symmetry axis and defining the position of the rotation axis, appears on the sinogram during the rotational motion of the slice. The regularity of these sinusoidal 


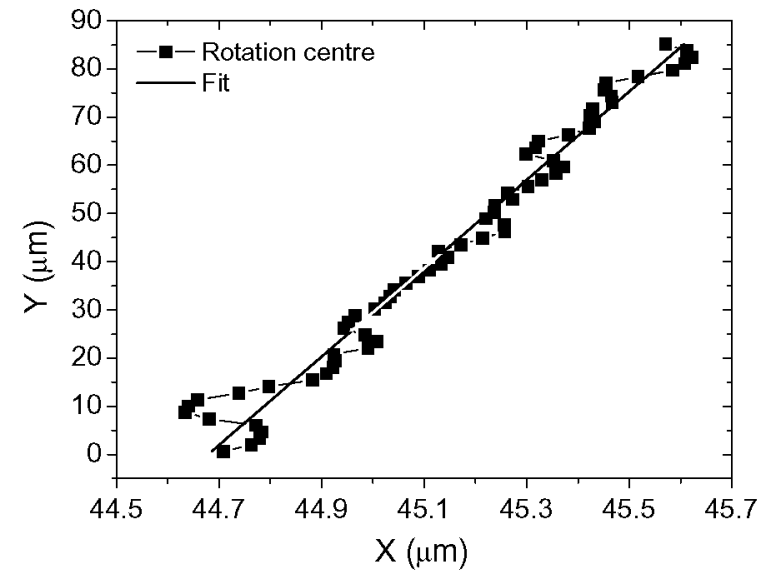

Fig. 5. Determination of the position of the rotation axis: the rotation centers calculated for each slice (black squares) are displayed. The solid line indicates the rotation axis obtained from these points.

patterns as visible in Fig. 4, is a good indication of the stability of the experimental setup during acquisition.

An important step in TomoRebuild reconstruction is the determination of the rotation axis of the step motor. This rotation axis is obviously close to the center of the sample, as it was adjusted experimentally, but not exactly in the middle of the sinogram. A least squared algorithm is used to determine the precise position of the rotation axis, as proposed by Azevedo et al. [18]. For this, the slice centre of mass is taken as a reference point. Its position is computed for each line of the sinogram and fitted by a least squares algorithm to obtain the equation of the corresponding sinusoid. It is possible to deduce the position of the experimental rotation centre from the sinusoid parameters. In Fig. 5, the position of the rotation centers is plotted for every slice. Then, a fit was performed to obtain the theoretical position of the rotation axis. These theoretical values are then used to perform the tomographic reconstruction. The discrepancies between the calculated rotation centres and the rotation axis were found to be very small $0.20 \pm 0.05 \mu \mathrm{m}$. This shows that the rotational motion of the sample is very stable. Moreover, the verticality of the axis is estimated to be inclined by $1.9 \pm 0.1^{\circ}$. The 64 tomographic slices were reconstructed separately, using the TomoRebuild code, and were numerically stacked to obtain the 3D images.

\section{Conclusions}

This preliminary study carried out on a glass microsphere reference sample shows for the first time the capability to perform $3 \mathrm{D}$
STIM-T experiments on the CENBG nanobeam line. The regular shape of the measured sinograms and the good alignment of the rotation centers obtained for every slice, are indicating the stability of the sample stage during the tomography experiment. Further experiments will require to include PIXE-T information to characterize the elemental distributions within the sample and to obtain a more accurate quantitative mass density determination. Such experiments are planned on organic samples.

\section{Acknowledgement}

This work has been supported by the European Community as an Integrating Activity "Support of Public and Industrial Research Using Ion Beam Technology (SPIRIT)" under EC contract no. 227012

\section{References}

[1] C. Habchi, D.T. Nguyen, Ph. Barberet, S. Incerti, Ph. Moretto, A. Sakellariou, H. Seznec, Nucl. Instr. and Meth. B 267 (2009) 2107.

[2] M. Rothermel, T. Reinert, T. Andrea, T. Butz, Nucl. Instr. and Meth. B 268 (2010) 2001.

[3] T. Satoh, M. Oikawa, T. Kamiya, Nucl. Instr. and Meth. B 267 (2009) 2125.

[4] A.E. Pontau, A.J. Antolak, D.H. Morse, A.A. Berkmoes, J.M. Brase, D.W. Heikkinen, H.E. Martz, I.D. Proctor, Nucl. Instr. and Meth. B 40 (1989) 646.

[5] R.M.S. Schofield, H.W. Lefevre, Nucl. Instr. and Meth. B 72 (1992) 104.

[6] Ph. Moretto, C. Michelet, S. Le Gallet, F. Rebillat, A. Guette, G. Vignoles, Nucl. Instr. and Meth. B 181 (2001) 238.

[7] T. Andrea, M. Rothermel, T. Butz, T. Reinert, Nucl. Instr. and Meth. B 267 (2009) 2098.

[8] A. Sakellariou, M. Cholewa, A. Saint, G.J.F. Legge, Nucl. Instr. and Meth. B 130 (1997) 253.

[9] A. Sakellariou, D.N. Jameieson, G.J.F. Legge, Nucl. Instr. and Meth. B 181 (2001) 211.

[10] T. Andrea, M. Rothermel, R. Werner, T. Butz, T. Reinert, Nucl. Instr. and Meth. B $268(2010) 1884$

[11] C. Habchi, P. Moretto, Nucl. Instr. Meth. B 150 (1999) 173.

[12] S. Incerti, C. Habchi, Ph. Moretto, J. Oliver, H. Seznec, Nucl. Instr. and Meth. B 249 (2006) 738.

[13] S. Incerti, Q Zhang, F. Andersson, Ph. Moretto, G.W. Grime, M. Merchant, D.T. Nguyen, C. Habchi, T. Pouthier, H. Seznec, Nucl. Instr. and Meth. B 260 (2007) 20.

[14] Ph. Barberet, S. Incerti, F. Andersson, F. Delalee, L. Serani, P. Moretto, Nucl. Instr. and Meth. B 267 (2009) 2003.

[15] Ph. Barberet, L. Daudin, N. Gordillo, S. Sorieul, M. Simon, H. Seznec, I. Idarraga, $\mathrm{S}$. Incerti, Ph. Moretto, Abstract submitted to this 12th International Conference on Nuclear Microprobe Technology and Applications (2010). This proceedings.

[16] http://www.brookhaven.co.uk/

[17] C. Michelet-Habchi, S. Incerti, P. Aguer, Ph. Barberet, E. Gontier, K. Grente, Ph. Moretto, D.T. Nguyen, A. Pouthier, T. Pouthier, F. Rebillat, R.W. Smith, Nucl. Instr. and Meth. B 231 (2005) 142.

[18] S.G. Azevedo, D.J. Schneberk, J.P. Fitch, H.E. Martz, IEEE Trans. Nucl. Sci. 37/4 (1990) 1525 